Apidologie, 1984, 15 (3), 303-314

\title{
COMPOSITION DE LA FRACTION LIPIDIQUE DE LA GELÉE DE LARVES D'ABEILLES REINES ET OUVRIÈRES (APIS MELLIFERA LIGUSTICA SPINOLA) EN FONCTION DE L'ÂGE DES LARVES
}

\author{
Giovanni LERCKER * Maria Adelaïde VECCHI ** Lucia PIANA **, \\ Antonio NANETTI ** et Anna Gloria SABATINI ** \\ :Dipartimento di Protezione e Valorizzazione Agro-Alimentare, \\ Università di Bologna (Italie). \\ * Istituto Nazionale di Apicoltura, Bologna (Italie).
}

\begin{abstract}
RESUMÉ
On a analysé les fractions lipidiques de séries d'échantillons de gelée royale (G.R.) et de gelée destinée aux larves d'ouvrières (G.O.) classées en fonction de l'âge des larves. On a comparé les compositions des esters méthyliques des acides organiques libres, obtenues par éluition gaz-chromatographique avec colonne à paquet (OV $2251,5 \%$ ). La composition quali-quantitative des deux aliments des larves a résulté être fort semblable au cours des trois premiers jours; durant la période entre le $3^{*}$ et le $5^{e}$ jour, la quantité totale de lipides et la répartition qualitative des acides organiques libres changent remarquablement.
\end{abstract}

\section{INTRODUCTION}

Plusieurs recherches ont été menées afin de déterminer la composition des aliments des larves et les mécanismes d'induction de la différenciation (Planta A., 1898, 1899 ; Elser R., 1929 ; Rhein W., 1933 ; Townsend G.F. and Lucas C.C., 1940 ; Haydak M.H., 1943 ; Habovsky J.E.J. et Shuel R.W., 1959 ; Patel N.G. et al., 1960 ; Patel N.G. et al., 1961 ; HaYdaK M.H., 1961 ; Jung-HoffmanN I., 1966, 1968 ; Weaver N. et al., 1968 ; Pain J., 1968 ; HaydaK M.H., 1968 ; Boch R. et al., 1979 ; BeEtsma J., 1979 ; Rembold H., 1980 ; Weiss K., 1980, 1981 ; Thrasyvoulou A.T. et al., 1983).

Cette étude a pour but d'établir la composition quali-quantitative de la fraction lipidique des aliments des larves femelles d'abeille en utilisant l'analyse gaz-chromatographique (LercKer G. et al., 1981) des extraits correspondants d'échantillons prélevés suivant des modes et des périodes permettant de localiser d'éventuelles relations entre la composition et : 
- l'âge et la caste des larves ;

- absence ou présence de la reine ;

- âge des abeilles nourrices ;

— conditions du milieu ;

c'est-à-dire des paramètres tout à fait importants pour le conditionnement de l'équilibre biologique de la famille tout entière.

\section{MATÉRIEL ET MÉTHODES}

Le prélèvement des échantillons s'est étalé sur deux années consécutives (1979-1980).

$\mathrm{Au}$ cours de la première année de recherches, en pleine saison (mois de juin), on a étudié une série d'échantillons de gelée royale (G.R. $\mathbf{n}^{*}$ 1) prélevés toutes les 24 heures de cellules en élevage artificiel, à partir du premier jour après le greffage jusqu'à l'operculation.

Le contenu de chaque cellule (sept pour chaque groupe d'âge) a été analysé individuellement afin de vérifier si la composition de la fraction lipidique était constante pour les larves de même âge.

La même année (mois de juillet) et par une technique analogue, on a conduit des recherches sur une série de prélèvements de gelée destinée aux larves d'ouvrières, divisées suivant leur âge (G.O.) ; les contenus des nombreuses cellules utilisées pour chaque groupe d'âge ont été mélangés en un seul échantillon. Pour tous les prélèvements de l'année suivante, on a appliqué la même méthode.

$\mathrm{Au}$ cours de la deuxième année de recherches, les prélèvements de G.R. et de G.O. ont été pris dans quatre ruches d'un deuxième rucher. En février-mars (juste après la reprise de la ponte) on a effectué deux séries de prélèvements dans des cellules d'ouvrière soignées par des insectes en hivernage.

Ensuite, au cours de la saison active (mai, juin et juillet), les prélèvements de G.R. et de G.O. ont continué sur trois ruches différentes (No. 4 à 6) dans lesquelles, à des moments différents, on induisait l'orphelinage et l'augmentation de l'âge des abeilles les plus jeunes de ces ruches. Ces prélèvements correspondent aux numéros 5 à 14. En résumé, les prélèvements de nourrissement larvaire d'ouvrière ont été effectués sur des colonies :

- à reine et ouvrières d'âge normal (séries 2,9 et 13);

- à reine et ouvrières vieilles (séries $3,4,6$ et 14);

- sans reine et à ouvrières d'âge normal (séries 5 et 8 ) ;

- sans reine et à ouvrières vieilles (séries 7 et 10 ).

La gelée royale a été prélevée de cellules royales élevées dans trois ruches (séries 1, 11 et 12).

D'autres séries d'échantillons ont été prises encore en pleine saison (mois de juillet) de cellules royales en élevage artificiel dans un troisième rucher (série 15) (*).

(*) Dans les expériences portant sur l'élevage de reines, on a utilisé des larves de 24 à 36 heures pour les greffes. Pour les larves d'ouvrières, l'âge a été déterminé visuellement, sur la base de leurs dimensions et de leur position; les larves ont été divisées en 5 groupes homogènes (d'après leurs dimensions); après marquage des cellules contenant des oufs on a vérifié, par comparaison si les cinq groupes correspondaient effectivement à des larves de 1, 2, 3, 4 et 5 jours d'âge. 
Pour les prélèvements de G.R. et de G.O., la méthode suivante a été utilisée : les barrettes porte-cellules ou les rayons de couvain étaient enlevés des ruches et portés immédiatement au laboratoire où l'on procédait à l'extraction des larves, à leur numération et à leur pesage. Le contenu des cellules était prélevé par aspiration immédiatement après à l'aide de micropipettes à bulle préalablement jaugées et conservées à $4{ }^{\circ} \mathrm{C}$.

La littérature précise que, au point de vue chimique, la fraction lipidique des nourrissements pour larves de reines et d'ouvrières (fig. 1) est formée de 85 à $90 \%$ d'acides organiques libres (Townsend G.F. and LuCAs C.C., 1940 ; Boch R. et al., 1979 ; LeRCKer et al., 1981), qui, étant 90 à $95 \%$ des acides organiques totaux (libres et combinés) (Lercker et al., 1982) peuvent être considérés comme un indice correct de la teneur totale en acides.

\section{Gelée RoYale}

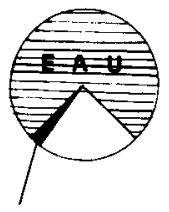

LIPIDES

\section{ACIDES}

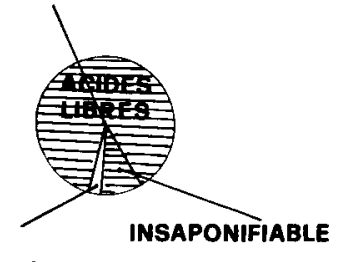

FIG. 1. - Composition de la gelée royale: eall et fraction lipidique

(TOWNSEND G.F. et LuCas C.C., 1940 ;

BACH R. et al., 1979; LeRCKER et al., 1981 ; LERCKER et al., 1982).

FIG. 1. - Composition of royal jelly : water and lipid fraction

(Townsend G.F. and Lucas C.C., 1940 ;

BACH R. et al., 1979; LeRCKER et al., 1981 ; LERCKER et al., 1982).

Comme les données précédentes ont été confirmées par les premières séries d'échantillons examinés, la teneur en acides libres a été considérée comme représentant la quantité totale de lipides du nourrissement.

Les gelées n'ayant pas une composition homogène (RHeIN W., 1933 ; JUNG-HoffMAN I., 1966), il a fallu utiliser la totalité de l'échantillon, qu'il provienne d'une seule cellule de reine ou d'un grand nombre de cellules d'ouvrières considérées ensemble.

$\mathrm{Au}$ moyen de petites quantités d'éther éthylique ajoutées à raison de $1: 5$ en poids/volume on a extrait la fraction lipidique jusqu'à épuisement de l'échantillon; le produit ainsi obtenu a été ensuite traité avec une solution de diazométhane en éther éthylique pour transformer en esters méthyliques les acides libres du résidu constituant les lipides totaux.

Pour trois séries d'échantillons de G.O. sur les onze examinées et pour une série de G.R., on a analysé la composition des acides libres et celle des acides totaux, obtenue après transestérification au méthanol-acide chlorhydrique des lipides correspondants. On a pu constater qu'en fonction de l'âge les taux d'acides organiques suivent la même courbe, les lipides étant identiques d'un point de vue qualitatif.

L'examen par chromatographie en phase gazeuse (préalablement mise au point sur colonne de $2 \mathrm{~m}$ à diamètre intérieur de $3 \mathrm{~mm}$, remplie de OV 225 à 1,5\%) des esters méthyliques ainsi obtenus a mis en évidence environ trente pics, dont certains correspondaient sans aucun doute à plusieurs substances (fig. 2).

L'identification de ces acides, qui ont déjà fait l'objet d'une étude (Lercker G. et al., 1981), a. confirmé l'existence de quatre classes de composés dans cette catégorie, plus précisément les 
acides gras. les acides dicarboxyliques. les mono- et les dihydroxydérivés. Pour les déterminations quantitatives, des corrections ont été faites sur la base des résultats gaz-chromatographiques, avec un détecteur à ionisation de flamme (F.I.D.), sur des composés étalon, standard ou de synthèse (LERCKER G. et al., 1982).

Acides totaux (e. m.)

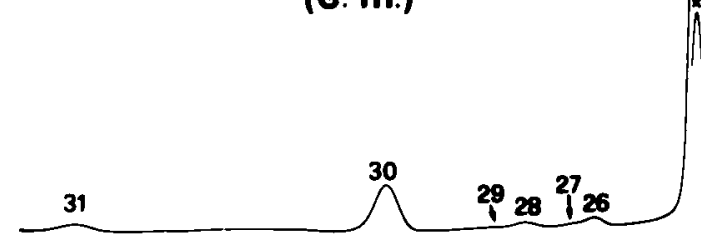

FIG. 2. - Distribution en chromatographie gazeuse des acides organiques (esters méthyliques) de la gelée royale et principaux acides identifiés

1 : dicarboxylique, 2 : monohydroxycarboxylique, 3 : méthyl-hésendioïque, 4 : non identifié, 5 : hydroxy-7 octanoïque, 6 : dicarboxylique, 7 : dicarboxylique, 8 : hydroxy-3 décanoïque et hydroxy-6 décanoïque, 9 : méthyl-octandioïque, $10:$ n-nonandiö̈que, 11 : hydroxy-8 octanoïque et p-hydroxybenzoïque, 12 : non identifié, 13 : méthyl-octendioïque, 14 : hydroxy-9 nonanoïque, 15 : m-benzodicarboxylique 16 : hydroxy-9 décanoïque, $17:$ n-décandiö̈que, $18:$ hydroxy-10 décanoïque, 19 : hydroxy-9 décène-2 rams ö̈que, 20 : palmitique, 21 : monohydroxycarboxylique, 22 : n-décendiö̈que, 23 : monohydroxycarboxylique, 24 : monohydroxycarboxylique, $25:$ hydroxy-10 décène-2 trans ö̈que, 26 : dicarboxylique, 27 : méthyl-tridécendiö̈que, 28 : hydroxy-11 décanoïque, 29 : hydroxy-11 dodécanoïque, 30 : octadécanoïque, 31 : dihydroxy-3,10 décanoïque.

FIG. 2. - Gas-chromatographic distribution of organic acids (methyl esters) of royal jelly. Identification of the main components and classes of components identified.

1 : dicarboxylic, 2 : monohydroxycarboxylic, 3 : methyl-hesendioic, 4 : not identified, 5 : 7-hydroxyoctanoic, 6 : dicarboxylic, 7 : dicarboxylic, $8:$ 3-hydroxydecanoic and 6-hydroxydecanoic, 9 : methyl-ocatandioic, $10:$ n-nonandioic, $11: 8$-hydroxyoctanoic and p-hydroxybenzoic, $12:$ not identified, 13 : methyl-octendioic, $14: 9$-hydroxynonanoic, $15:$ m-benzodicarboxylic, $16: 9$-hydroxydecanoic, $17:$ n-decandioic, $18:$ 10-hydroxydecanoic, $19: 9$-hydroxy,2-trans-decenoic, $20:$ palmitic, 21 : monohydroxycarboxylic, 22 : n-decendioic, 23 : monohydroxycarboxylic, 24 : monohydroxycarboxylic, $25:$ 10-hydroxy,2-trans-decenoic, 26 : dicarboxylic, $27:$ methyl-tridecendioic, $28: 11$-hydroxyndecanoic, 29 : meth 11-hydroxydodecanoic, 30 : octadecenoic, 31 : 3.10-dihydroxydecanoic.

\section{RÉSULTATS}

De même que pour l'étude précédente (Lercker G., 1982), on a observé une certaine variabilité quantitative des deux principales composantes, l'acide hydroxy10 décène-2 oïque (pic 25 de la figure 2), et l'acide hydroxy-10 décanoïque (pic 18 sur la figure 2), ct la similitude des structures moléculaires des principales composantes appartenant aux différentes classes de substances organiques. Cela laisse présumer que certaines composantes, très probablement celles dont les taux sont les plus faibles, sont des intermédiaires dans la biosynthèse des autres (WEavliz N. et al., 1968 ; LeRCKER G. et al., 1981). 
Ces deux aspects nous ont amené à choisir comme critère d'évaluation des résultats non seulement la teneur en acides organiques libres (considérée comme étant proportionnelle à celle des lipides) mais aussi le rapport entre le taux d'acide hydroxy-10 décène-2 oïque et celui d'acide hydroxy-10 décanoïque (notée $\mathrm{R}_{1}$ ), ainsi que le rapport entre la somme des taux des acides mono-hydroxyliques et celle des taux des acides dicarboxyliques (notée $R_{2}$ ).

Les résultats des examens effectués sur le contenu intégral des différentes cellules royales, sept pour chaque classe d'âge larvaire, ont été soumis à l'analyse statistique en vue de déterminer la variabilité de la fraction lipidique. On a pu constater que dans tous les échantillons du même groupe d'âge, la composition des acides organiques était très constante, et que pour les taux moyens, calculés en fonction de l'âge larvaire, l'analogie était raisonnable $\left({ }^{*}\right)$.

Les déterminations effectuées sur les échantillons de nourrissement pour les larves d'ouvrières, un par groupe d'âge considéré, ont révélé un comportement tout à fait différent de celui de la gelée royale.
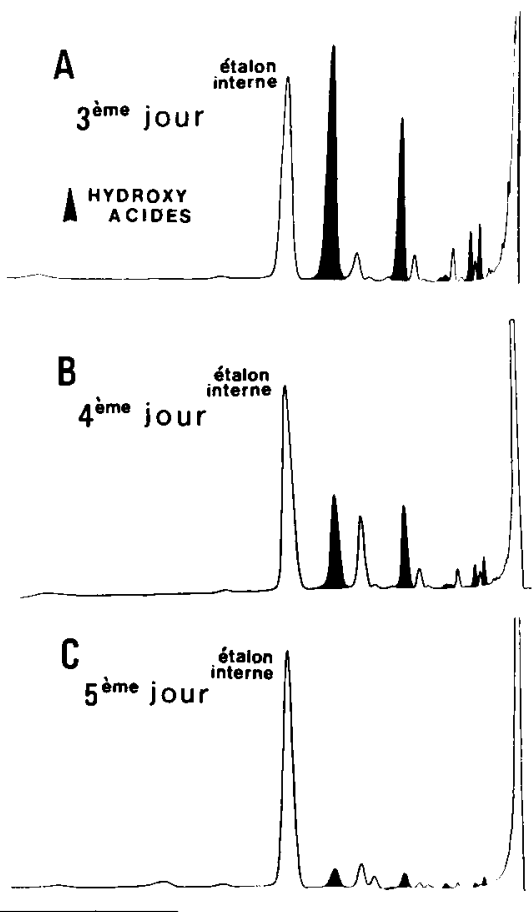

\footnotetext{
Fig. 3. - Tracés en chromatographie gazeuse des acides libres des nourrissements larvaires d'ouvrières après : A : trois jours de vie, $B$ : quatre jours de vie et $C:$ cinq jours de vie.

FIG. 3. - Gas-chromatographic traces of free acids in the food of worker larvae after:

$A$ : three days, $B$ : four days and $C$ : five days.
}

(*) La quantité de gelée obtenue de chaque cellule royale a varié de $30 \mathrm{mg}$ pour les larves les plus jeunes placées dans les conditions les plus défavorables (vieilles nourrices) à 400-450 mg pour les larves de quatre jours des colonies fortes.

En ce qui concerne les cellules d'ouvrières, la quantité de G.O. a oscillé, dans les différents prélèvements, de quelques dixièmes de $\mathrm{mg}$ par cellule pour les larves d'un jour à un maximum de 8 à $17 \mathrm{mg}$ par cellule pour les larves de 3 à 4 jours; à l'exception d'un cas, pour lequel, dans une colonie orpheline, on a relevé une quantité de G.O. relativement plus importante. 
En effet, si le profil gaz-chromatographique au cours des 3 premiers jours est le même, on peut constater une réduction de la teneur pourcent des hydroxyacides à partir du $4^{\circ}$ mais surtout du $5^{\circ}$ jour larvaire, ce qui s'accompagne également d'une réduction de la teneur totale en acides organiques libres, et avec une diminution légère des acides dicarboxyliques seulement, par rapport à la G.O. pour les larves jeunes (Fig. 3).
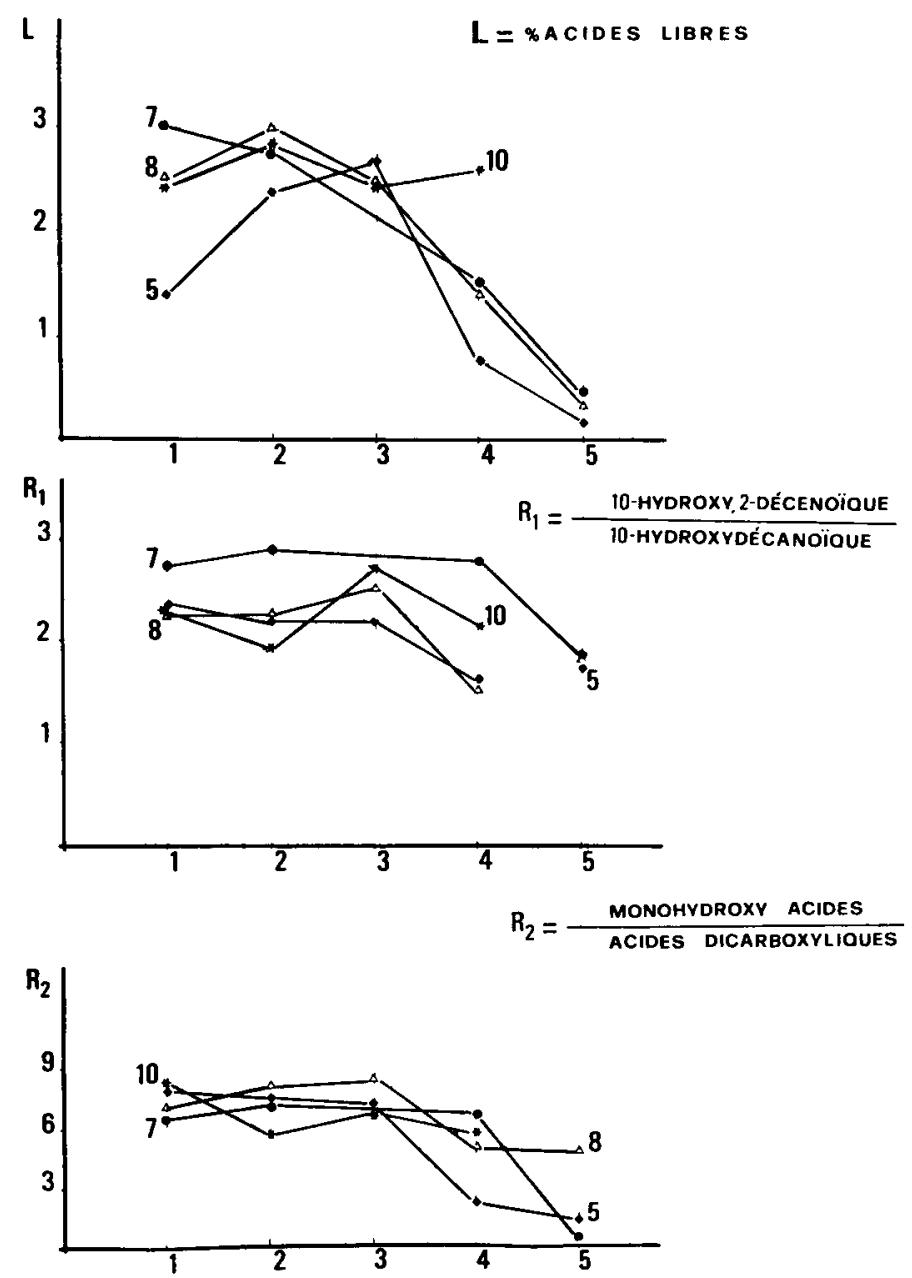

FIG. 4. - Evolution des paramètres choisis (taux des acides libres dans la matière fraiche, $R_{1}$ et $R_{2}$ )

Séries 5 et 8 : G.O., sans reine et à ouvrières d'âge normal. Séries 7 et 10 : G.O., sans reine et à ouvrières vieilles.

FIG. 4. - Evolution of chosen parameters

(free acids as a \% of the royal jelly, $R_{1}, R_{2}$ )

Numbers 5 and 8 : G.O., quenless, normal workers. Numbers 7 and 10 : G.O., queenless older workers. 
Par rapport à la teneur totale en acides libres, les acides dicarboxyliques (surtout le n-décènedioïque) deviennent les acides principaux de la G.O. pour les larves de 5 jours d'âge, ce qui est prouvé par la diminution du rapport $\mathbf{R}_{2}$ (hydroxyacides-acides dicarboxyliques), qui tombe à moins de l'unité pour quelques cas (Fig. 4 et 5).
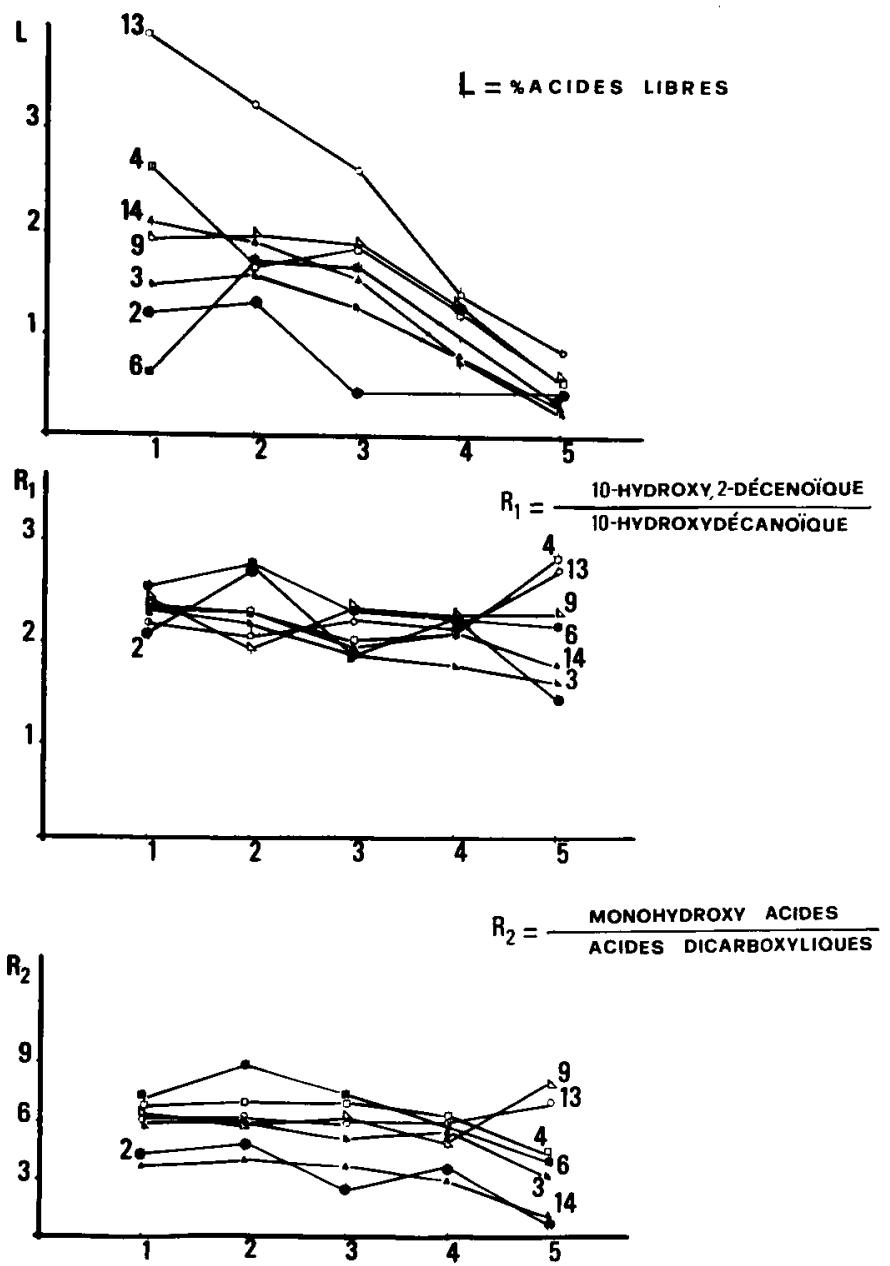

FiG. 5. - Evolution des paramètres choisis (taux des acides libres dans la matière fraîche, $R_{1}$ et $R_{2}$ )

Séries 2,9 et 13 : G.O., à reine et ouvrières d'âge normal. Séries 34,6 et 14 : G.O., à reine et ouvrières vieilles.

FIG. 5. - Evolution of chosen parameters

(free acids as $a \%$ of the royal jelly, $R_{1}, R_{2}$ )

Numbers 2, 9 and 13 : G.O., queenright, normal workers. Numbers 3, 4, 6 and 14 : G.O., queenright older workers. 


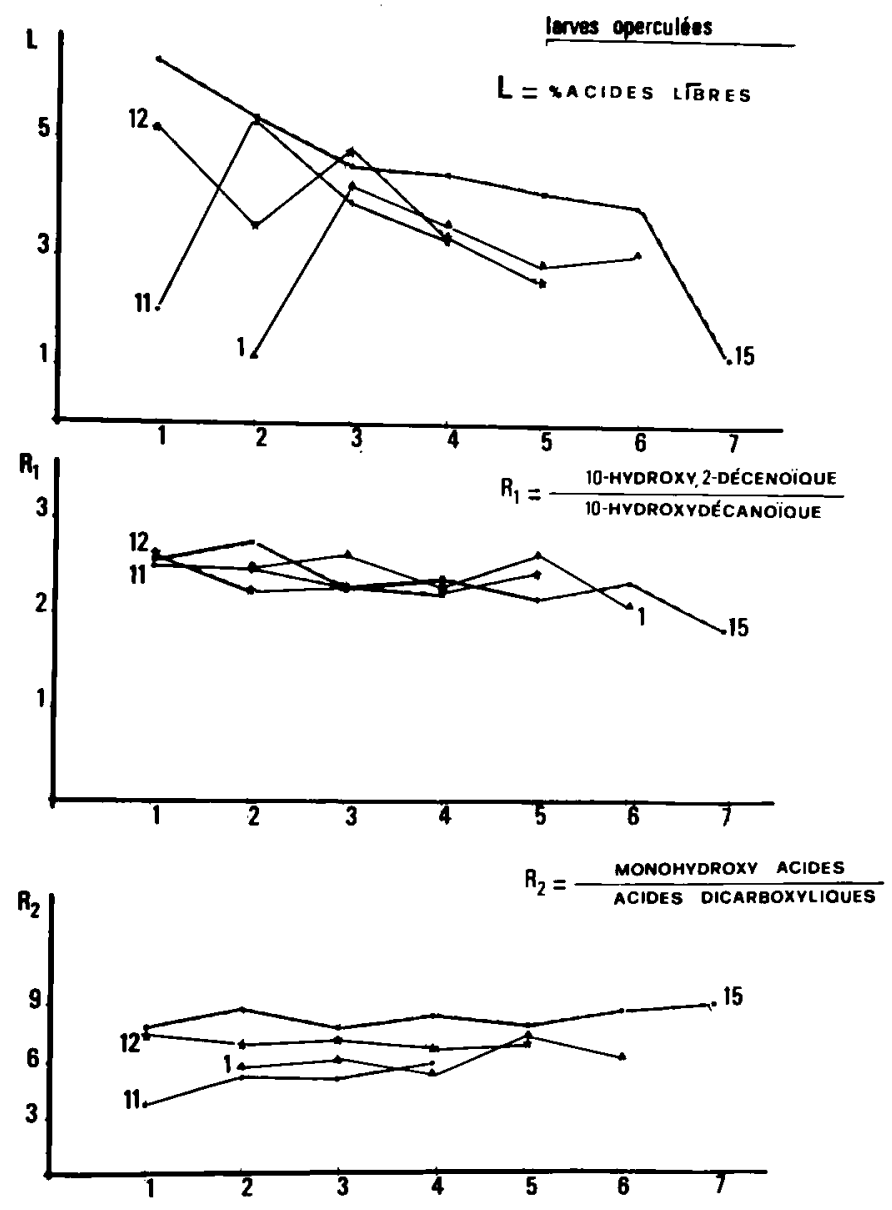

FIG. 6. - Evolution des paramètres choisis (taux des acides libres dans la matière fraiche, $R_{I}$ et $R_{2}$ )

Séries 1, 11 et 12, G.R., élevage naturel. Série 15, G.R., élevage artificiel.

Fig. 6. - Evolution of chosen parameters (free acids as a \% of the royal jelly, $R_{1}, R_{2}$ )

Numbers 1, 11 and 12 : G.R., normal queen cells. Numbers $15:$ G.R., artificially-reared queen cells.

L'étude des acides liés permet également de mettre en évidence les différences du profil des acides organiques de la G.O. les quatrième et cinquième jours.

Pour ce qui est de la teneur en acides libres (lipides) (Fig. 4, 5 et 6), par rapport à l'âge des larves, on a pu faire les observations suivantes: 
- au cours des premiers jours la variabilité entre les différentes séries de prélèvements est élevée, mais 'elle diminue considérablement après le troisième jour ;

- dans le nourrissement des larves d'ouvrières le taux des acides libres baisse à mesure que la larve vieillit ;

- l'évolution du taux des acides libres ne semble pas être affectée par les conditions de milieu, ni par les facteurs internes de la colonie.

Le rapport $R_{1}$ (Fig. 4, 5 et 6 ) entre l'acide hydroxy-10 décène-2 oïque et l'acide hydroxy-10 décanoïque est assez constant, en particulier pour la gelée royale (Fig. 6).

Par contre, le rapport $\mathrm{R}_{2}$ (acides hydroxyliques/acides dicarboxyliques) révèle une situation différente; dans les gelées destinées aux larves d'ouvrières de presque toutes les séries d'échantillons, il diminue de manière appréciable entre les quatrième et cinquième jours d'âge (Fig. 4, 5 et 6). Cette baisse semble être encore plus nette dans les colonies où les abeilles sont vieilles; elle n'a pas été remarquée dans le cas de la gelée royale.

Ces faits semblent moins nets dans le cas des colonies à reine (Fig. 5) que dans celui des colonies orphelines.

En ce qui concerne la gelée royale, l'évolution de la teneur en acides libres et des paramètres $R_{1}$ et $R_{2}$ (Fig. 6), s'est montrée assez constante ; pour les acides libres et $R_{2}$ les valeurs déterminées étaient souvent plus élevées que dans la gelée d'ouvrière.

\section{CONCLUSIONS}

Les différences observées entre les deux types de nourrissements, compte tenu de l'âge des larves auxquelles ils étaient destinés, des conditions du milieu et de la situation de la colonie, ont permis de tirer les conclusions suivantes :

1. Les lipides des deux nourrissements sont constitués principalement d'acides libres, dont la composition relative quantitative et qualitative durant les trois premiers jours est la même dans ses grandes lignes, tout en ayant des totaux différents. Une variabilité élevée de la teneur en lipides des deux types de nourrissement a été enregistrée durant les premiers jours de vie des larves.

2. Les quatrième et cinquième jours de vie, les larves d'ouvrières reçoivent un nourrissement différent, en ce qui concerne tant la teneur en lipides que la composition des acides, particulièrement pauvre en acides hydroxyliques. On pourrait donc supposer que ces composantes jouent un rôle particulier dans le développement de la larve au cours des phases successives. 
3. Les conditions de milieu (saison et donc disponibilités alimentaires) et l'état de la colonie (présence ou absence de la reine et âge des nourrices) ne semblent pas affecter la teneur en lipides et la distribution des acides, à l'exception d'une baisse marquée du rapport $R_{\text {¿. }}$, observée dans les gelées des nourrices des colonies orphelines ou dont les abeilles sont vieilles.

Reçu pour publication en mars 1984. Accepté pour publication en juin 1984.

\section{SUMMARY}

\section{COMPOSITION OF THE LIPID FRACTION IN THE JELLY OF THE QUEEN BEE AND WORKER BEE LARVAE (APIS MELLIFERA LIGUSTICA SPINOLA) IN RELATION TO AGE}

An experiment was undertaken with the strict object of determining the composition and the qualitative aspects of the lipid fraction in the food destined for female bee larvae (Apis mellifera ligustica Spinola) using chromatographic methods (fig. 2).

Samples of royal jelly (G.R.) were collected from artificial breeding combs every 24 hours from grafting until capping. Seven cells of each age group were taken. Worker jelly (G.O.) was also collected from cells of larvae of different ages. Four colony types were sampled for G.O. as follows : 1) queenright colonies with normal workers; 2) queenright colonies with older workers; 3) queenless colonies with normal workers; 4) queenless colonies with older workers. G.O. was also sampled from these colony types at different times of the year.

Although there was an enormous difference in the quantity of jellies supplied to larvae of the two castes the qualitative composition of the free acids contained in the G.O. was the same as that in the G.R. until the 4th day of larval age. After this time the composition of G.O. changes considerably (fig. 3 ).

For each series of jelly samples analyzed, the following were compared in relation to the age of the larvae:

a) the content of free acids (proportional to the $\%$ of lipids); nents) :

b) the ratio $\left(\mathrm{R}_{1}\right)$ of 10 -hydroxy, 2 -decenoic acid to 10-hydroxydecanoic acid (principal compo-

c) the ratio $\left(\mathrm{R}_{2}\right)$ of the set of monohydroxy acids to the dicarboxylic acids (the two principal sets of compounds present).

For G.R. the values for $\%$ of lipids and $R_{1}$ and $R_{2}$ remained constant throughout larval development (fig. 6) ; the values for free acids and $R_{2}$ were often found to be higher than in G.O.

The pattern for \% lipids in G.O. (clearly diminishing with time) did not appear to be influenced by environmental conditions such as season and availability of food sources, nor by internal conditions such as queenlessness and age of the workers.

Again for G.O. (fig. 4 and 5) while the ratio $R_{1}$ was reasonably constant the ratio $R_{2}$, as well as the \% lipids, tended to diminish between the 4th and the 5th day of larval life. This diminution was more pronouned in hives with older adult worker bees and was not observed in the royal jelly (fig. 6). With regard to the total content of free acids, carboxylic acids (especially the n-decendioic acid) become the main acids of G.O. for 5 days old larvae. 


\title{
ZUSAMMENFASSUNG
}

\author{
ZUSAMMENSETZUNG DER LIPIDFRAKTION DES WEISEL- \\ UND ARBEITERINNENFUTTERSAFTES VON APIS MELLIFERA LIGUSTICA \\ IN BEZUG AUF DAS LARVENALTER
}

Es wurde ein Versuch mit dem Ziel unternommen, die Zusammensetzung und die qualitativen Aspekte der Lipidfraktion in der Nahrung für die weiblichen Bienenlarven (Apis mellifera ligustica Spinola) zu bestimmen. Zu diesem Zweck wurde die Methode der Gas-Chromatographic angewandt (Fig. 2).

Es wurden Stichproben des Weiselfuttersaftes (G.R.) analysiert, der aus Zellen von künstlichen Zuchtserien (sieben Zellen für jede Altersgruppe, vom ersten Tag nach dem Umlarven bis zur Verdeckelung) alle 24 Stunden entnommen wurde. In derselben Zeitperiode und mit analoger Technik wurden Probenreihen von Arbeiterinnenfuttersaft (G.O.) untersucht, und zwar je eine Stichprobe aus einer größeren Anzahl von Zellen für jedes Alter.

Bei jeder Versuchsreihe wurden folgende Parameter untersucht :

a) der Gehalt an freien Säuren (der sehr nahe bei dem Gesamtgehalt an Lipiden licgt);

b) das Verhältnis $\mathbf{R}_{1}$ zwischen 10-Hydroxy-2-Decanol- und 10-Hydroxydecanolsäure (den Hauptkomponenten der Lipidfraktion) ;

c) das Verhältnis $R_{2}$ zwischen der Gesamtheit der Hydroxyderitvatsïurcı und der Dicarbonsäuren (den beiden Hauptklassen der vorhandenen Lipide);

in Bezug auf das Larvenalter.

Abgesehen von den außerordentlich großen quantitativen Unterschieden in dem für die Larven der beiden Kasten getieferten Futtersaft ist die qualitative Zusammensetzung der freien Säuren in dem G.O. dieselbe wie die im analysierten G.R. wenigstens bis zum 4. Larventag. Danach ändert sich im G.O. die Zusımmensetzung bedeutend (Fig. 3).

Beim G.R. bleiben der Gehalt an freien Säuren und die Parameter $R_{1}$ und $R_{2}$ relativ konstant (Fig. 6) ; beim Gehalt an freien Säuren und bei $R_{22}$ liegt der festgestellte Wert oft über dem für G.O.

Beim G.O. (Fig. 4 und 5) bleibt das Verhältnis $R_{1}$ ziemlich konstant, während das Verhältnis $R_{2}$ (Hydroxysäuren/Dicarbonsäuren) in fast allen Versuchsreihen eine andere Tendenz zeigt : es nimmt zwischen dem 4. and 5. Tag des Larvenalters bemerkenswert ab (Fig. 4 und 5).

Diese Abnahme scheint besonders deutlich bei Völkern mit alten Bienen zu sein, bei G.R. wurde sie nie festgestellt (Fig, 6).

Die Entwicklung des Gehalts an freien Säuren im G.O. (deutliche Abnahme entsprechend dem Ablauf der Tage) scheint weder von den Umweltbedingungen (wie Jahreszeit und Trachtverhältnisse) noch von Innenbedingungen (wie Weisellosigkeit oder Weiselrichtigkeit oder dem Alter der Ammenbienen) abzuhängen.

\section{BIBLIOGRAPHE}

BeEtSMA J., 1979. - The process of queen-worker differentiation in the honeybee. Bee World, 60, 24-39.

Boch R., Shearer D.A., Shuel R.W., 1979. - Octanoic and other volatile acids in the mandibular glands of the honeybee and in royal jelly. J. apic. Res., 18, 250-252.

Elser E., 1929. - Die chemische Zusammensetzung der Nahrungstoffe der Biene. Märkische Bienen Ztg. 19, 201-215, 232-235, 248-252. 
HabowSKY J.E.J., ShUEl R.W., 1959. - Separation of the protein constituents of the larval diets of the honey-bee by continuous paper electrophoresis. Can. J. Zool., 37, 957-964.

HaydaK M.H., 1943. - Larval food and development of castes in the honey-bee. J. econ. Entomol., 36, $778-792$.

HAYDAK M.H., 1961. - The changes in the vitamin content of royal jelly produced by nurse bees of various ages in confinement. Bee World, 42, 57-59.

Haydak M.H., 1968. - Nutrition des larves d'abeilles. In : Traité de biologie de l'abeille, R. Chauvin éd., vol. 1. Paris, Masson, 302-333.

Jung-HoffmanN I. 1966. - Die Determination von Königin und Arbeiterin der Honigbiene. Z. Bienenforsch., 8, 296-322.

Jung-Hoffmann I., 1968. — Développement des larves. In : Traité de biologie de l'abeille, R. Chauvin éd., vol. 1. Paris, Masson, 69-99.

Lercker G., Capella P., Conte L.S., Ruini F., Giordani G., 1981. - The components of Royal Jelly. Note I. Identification of the organic acids. Lipids, 16, 912-919.

Lercker G., Capelta P., Conte L.S., Ruini F., Giordani G., 1982. - The components of Royal Jelly. Note II. The lipid fraction, hydrocarbons and sterols. J. apic. Res., 21 (3), 178-184.

PAIN J., 1968. - Le facteur trophique dans le déterminisme des castes. In : Traité de biologie de l'abeille, R. Chauvin éd., vol. 1. Paris, Masson, 45-67.

Patel N.G., Haydak M.H. Gochnauer T.A., 1960. - Electrophoretic components of the proteins in honey-bee larval food. Nature, 186, 633-634.

Patel N.G., Haydak M.H., Lovell R., 1961. - Chromatographic investigation of the methyl alcohol/chlorophorm soluble constituents of the honey-bee larval food. Nature, 191, 362-363.

Planta A. von, 1868. - Über den Futtersaft der Bienen. Z. Physiol. Chem., 12, 327-354.

Planta A. von, 1889. - Über den Futtersaft der Bienen. Z. Physiol. Chem., 13, 552-561.

REMBold H., 1980. - Der Weiselfuttersaft. In : Königinnenzucht biologische Grundlagen und technische Anleitungen, Apimondia, Bukarest, 19-24.

RHein A. von, 1933. - Uber die Entstehung des weiblichen Dimorphismus in Bienenstaate. Wilhelm Roux' Arch. Entwicklungs Mech. Organ., 129, 601-665.

Thrasyvoulou A.T., Collison C.H., Benton A.W., 1983. - Electrophoretic patterns of watersoluble proteins of honeybee larval food. J. apic. Res., 22 (3), 142-145.

Townsend G.F., Lucas C.C., 1940. - The chemical nature of royal jelly. Biochem. J., 34, 1155-1162.

Weaver N., Johnston N.C., Benjamin R., Law J.H., 1968. - Novel Fatty Acids From the Royal Jelly of Honeybee (Apis mellifera L.). Lipids, 3, 535-538.

WeIss K., 1980. - Über die Entstehung der weiblichen Kasten im Bienenvolk. In : Königinnenzucht biologische Grundlagen und iechnische Anleitungen, Apimondia, Bukarest, 25-44.

Weiss K., 1980. - Der Einfluss von Aufzuchtfaktoren auf die Entwicklung der Königinnen. In : Königinnenzucht biologische Grundlagen und technische Anleitungen, Apimondia, Bukarest, 65-134.

Weiss K., 1981, - Geheimnis der Königinnenentstehung. Imkerfreund, 36, extra, 1-4-81. 